Supporting Information for:

A Simple Molecular Rotor for Defining Nucleoside Environment within a DNA Aptamer-Protein Complex

Thomas Z. Cservenyi, Abigail J. Van Riesen, Florence D. Berger, Ahmed Desoky and Richard A. Manderville*

Table of Contents:

1. Figure S1. CD spectra of ${ }^{\text {Fur }}$ dU-mTBA duplexes and GQs $\quad$ S2

2. Figure S2. Normalized emission responses of mTBA to [protein] S3

3. Synthesis of ${ }^{\text {Fur }}$ dU-phosphoramidite $\quad S 4$

3. Figures S3-S6. NMR spectra of synthetic compounds S6

4. Table S1. MS data of ${ }^{\mathrm{Fur}} \mathrm{dU}$ mTBA strands $\quad S 8$

5. Figure S7. Representative ESI ${ }^{-}$MS Spectrum of ${ }^{\text {Fur }}$ dU-mTBA $\quad$ S9 


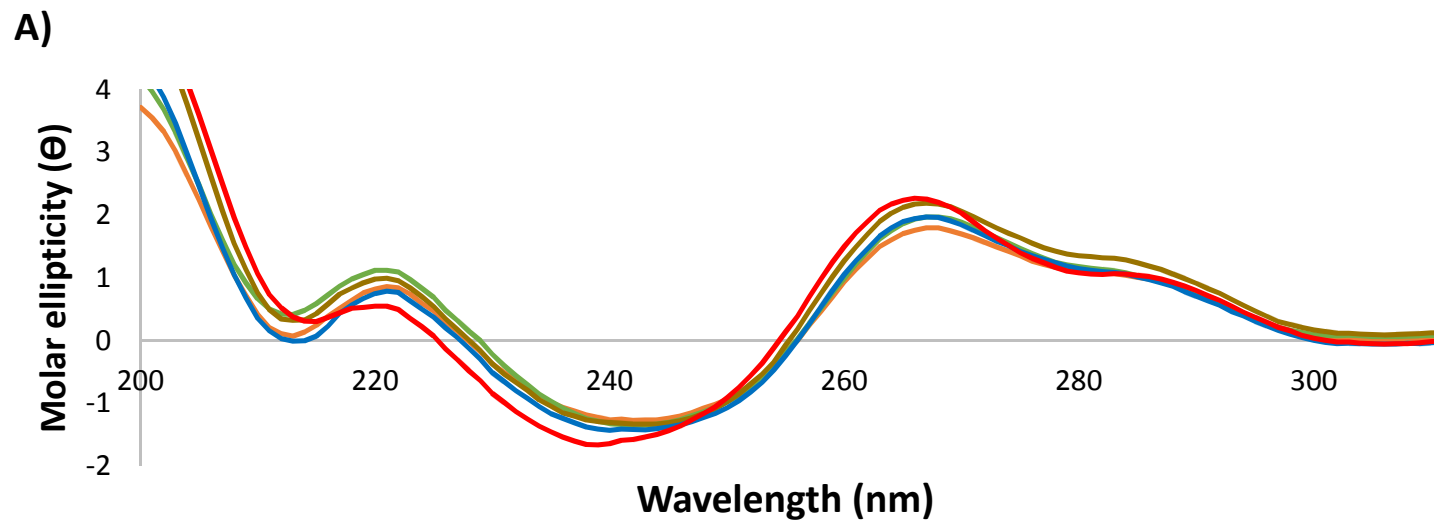

B)

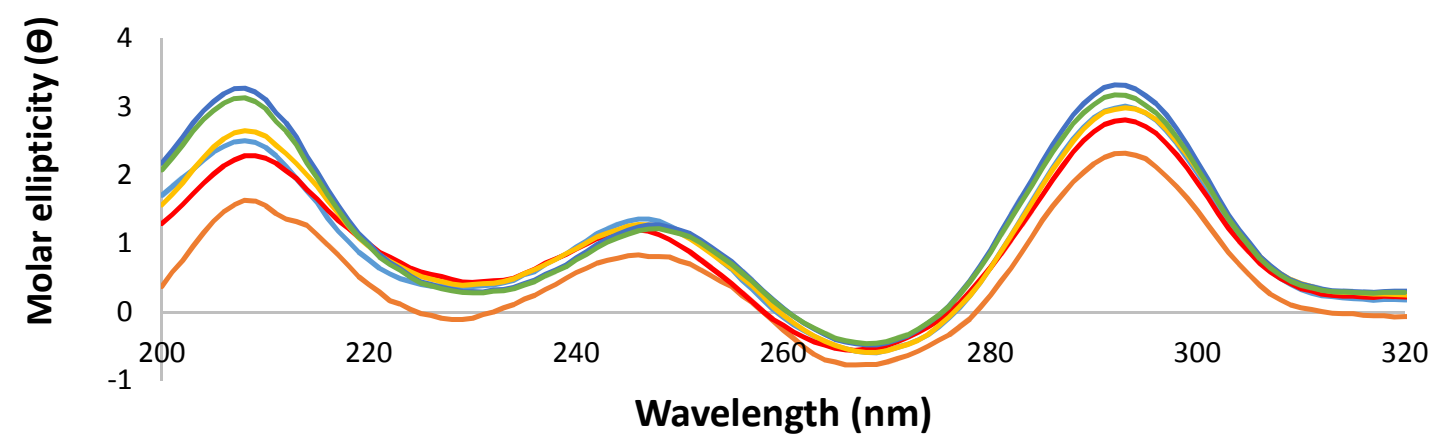

Figure. S1 Circular dichroism spectra of Fur dU-mTBA $(6 \mu \mathrm{M})$ recorded at $10^{\circ} \mathrm{C}$. A) Duplexes with 1 equiv. complementary strand in $100 \mathrm{mM} \mathrm{HNa}_{2} \mathrm{PO}_{4}$ buffer $\mathrm{pH} 7.0,100$ $\mathrm{mM} \mathrm{NaCl}$. B) GQs in $100 \mathrm{mM} \mathrm{HK}{ }_{2} \mathrm{PO}_{4}$ buffer pH 7.0, $100 \mathrm{mM} \mathrm{KCl}$; ${ }^{\text {Fur }} \mathrm{dU}-3$ (red), ${ }^{\text {Fur dU-4 }}$

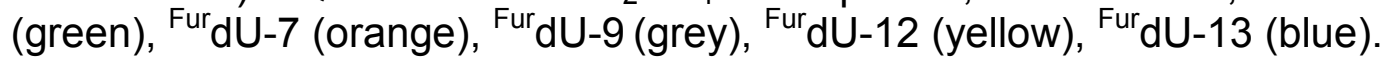



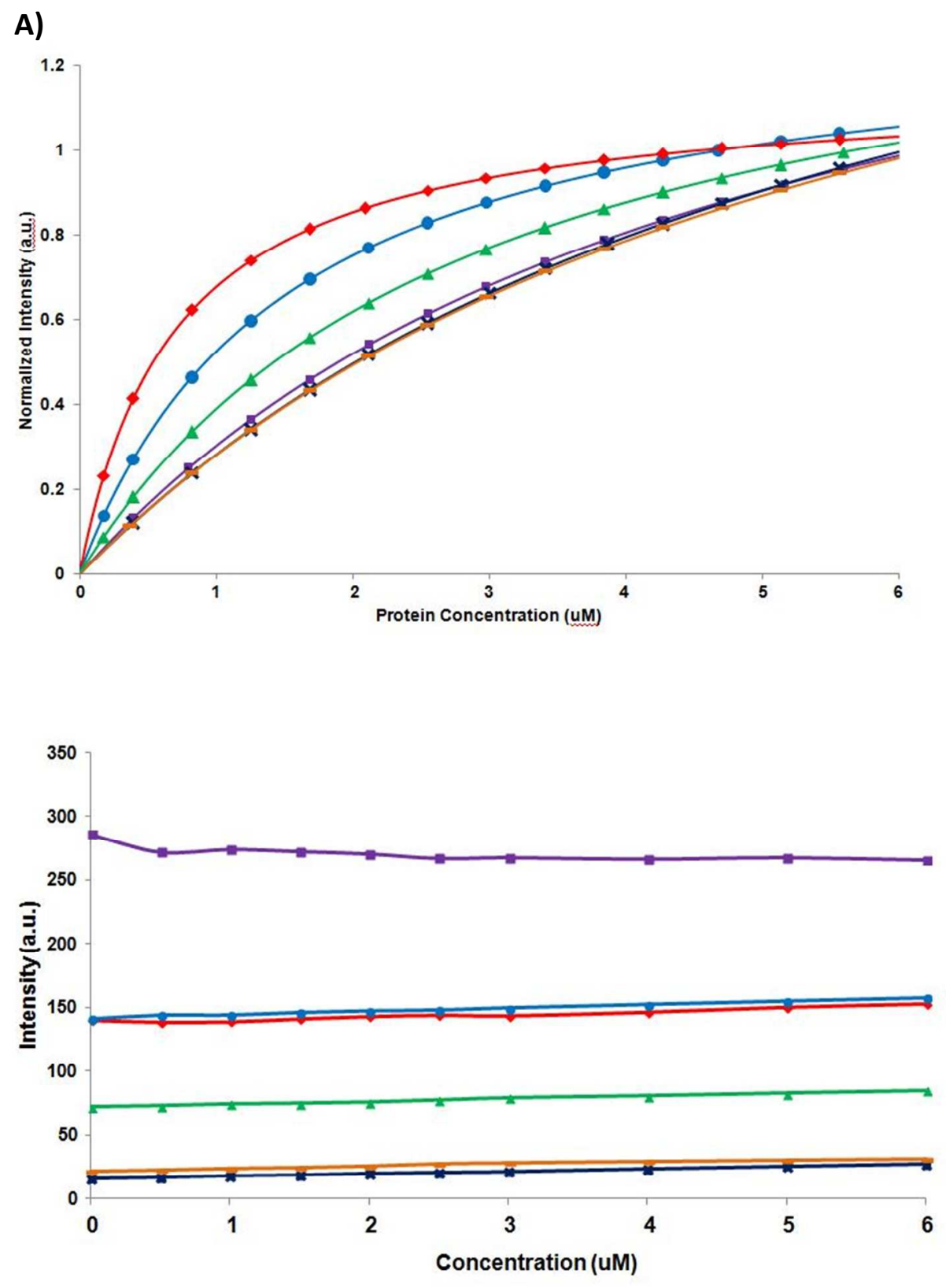

Figure S2. A) Normalized emission intensity of mTBA upon successive addition of thrombin, B) emission intensity changes upon successive addition of BSA; FurdU-3 (red),

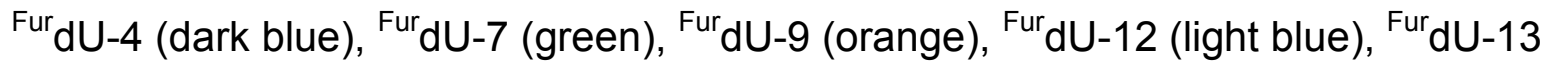
(purple). 


\section{Synthesis of FurdU-Phosphoramidite}

The Fur dU-phosphoramidte was prepared according to the literature procedure (Greco, N. J., and Tor, Y. (2007) Synthesis and site-specific incorporation of a simple fluorescent pyrimidine. Nat. Protoc. 2, 305-316).

(i) Synthesis of 5-(fur-2-yl)-2'-deoxyuridine ( $\left.{ }^{\mathrm{Fur}} \mathrm{dU}\right)$

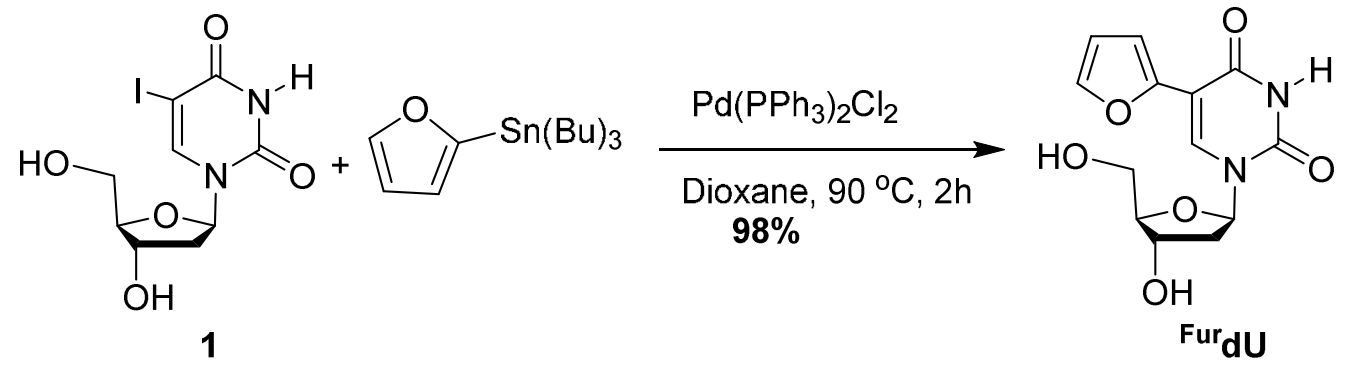

In a flame dried 2-neck $100-\mathrm{mL}$ flask, a mixture of 5-iodo-2'-deoxyuridine $(3 \mathrm{~g}, 8.47$ $\mathrm{mmol}$ ) and dichlorobis(triphenylphosphine)Pd(II) $(0.12 \mathrm{~g}, 0.17 \mathrm{mmol})$ under nitrogen atmosphere in anhydrous dioxane $(60 \mathrm{ml})$ was added 2-(tributylstannyl)furan $(11.5 \mathrm{ml}$, $36.43 \mathrm{mmol}$ ) via a syringe. The suspension was refluxed at $90{ }^{\circ} \mathrm{C}$ under nitrogen atmosphere for $2 \mathrm{~h}$, where TLC $\left(10 \% \mathrm{MeOH}\right.$ in $\left.\mathrm{CHCl}_{3}\right)$ indicates that the reaction is complete. The reaction mixture was cooled to room temperature and filtered through celite 545. The celite was washed with $150 \mathrm{~mL}$ methanol; the solvent was removed under reduced pressure where the resulting oil was triturated with hexanes $(3 \times 100 \mathrm{~mL})$ to produce a solid. The resulting solid was taken up in a minimum of hot solvent $(1 / 1$ methanol/chloroform) and precipitated from hexanes. The formed precipitate was filtered, washed with hexane then dried under vacuum to give the desired product as white solid (2.48 g, $98 \%$ yield). ${ }^{1} \mathrm{H}$ NMR (300 MHz, DMSO- $\left.d_{6}\right): \delta 11.62(\mathrm{~s}, \mathrm{NH}, 1 \mathrm{H})$, $8.32(\mathrm{~s}, 1 \mathrm{H}), 7.60(\mathrm{~s}, 1 \mathrm{H}), 6.84(\mathrm{~d}, J=2.8 \mathrm{~Hz}, 1 \mathrm{H}), 6.51(\mathrm{~m}, 1 \mathrm{H}), 6.22(\mathrm{t}, J=6.42 \mathrm{~Hz}$, 1H), $5.26(\mathrm{~d}, J=4.4 \mathrm{~Hz}, 1 \mathrm{H}), 5.08(\mathrm{t}, J=4.4 \mathrm{~Hz}, 1 \mathrm{H}), 4.27(\mathrm{~m}, 1 \mathrm{H}), 3.82(\mathrm{~m}, 1 \mathrm{H}), 3.59$ $(\mathrm{m}, 2 \mathrm{H}), 2.16(\mathrm{t}, \mathrm{J}=5.4 \mathrm{~Hz}, 2 \mathrm{H})$. 
(ii) Synthesis of 5'-dimethoxytrityl-5-(fur-2-yl)-2'-deoxyuridine (2)<smiles>COc1ccc(C(Cl)(c2ccccc2)c2ccc(OC)cc2)cc1</smiles>

A mixture of Fur dU (0.7 g, $2.37 \mathrm{mmol}), \operatorname{DMTr}-\mathrm{Cl}(0.96 \mathrm{~g}, 2.85 \mathrm{mmol})$ and DMAP (0.08 g, $0.65 \mathrm{~mol})$ in anhydrous pyridine $(20 \mathrm{ml})$ was stirred at room temperature under nitrogen atmosphere for $16 \mathrm{~h}$. The solvent was evaporated under reduced pressure. The product was purified by flash column chromatography $\left(\mathrm{CHCl}_{3}: \mathrm{MeOH} ; \mathrm{Et}_{3} \mathrm{~N} ; 97: 2\right.$ : 1). The desired product was isolated as white foam (1.06 g, 75\% yield). ${ }^{1} \mathrm{H}$ NMR (300 MHz, $\left.\mathrm{CDCl}_{3}\right): \delta 8.16(\mathrm{~s}, 1 \mathrm{H}), 7.43(\mathrm{bd}, 2 \mathrm{H}), 7.33--7.30(\mathrm{~m}, 4 \mathrm{H}), 7.21-7.12(\mathrm{~m}, 4 \mathrm{H}), 6.95(\mathrm{~d}$, $J=3 \mathrm{~Hz}, 1 \mathrm{H}), 6.77-6.72(\mathrm{~m}, 5 \mathrm{H}), 6.41(\mathrm{t}, J=7.03 \mathrm{~Hz}, 1 \mathrm{H}), 6.26(\mathrm{~m}, 1 \mathrm{H}), 4.4(\mathrm{~m}, 1 \mathrm{H})$, $3.71(\mathrm{~s}, 6 \mathrm{H}), 3.5(\mathrm{~m}, 1 \mathrm{H}), 3.32(\mathrm{~m}, 1 \mathrm{H}), 2.51(\mathrm{~m}, 1 \mathrm{H}), 2.33-2.24(\mathrm{~m}, 1 \mathrm{H})$.

(iii) 3'-(2-cyanoethyl)-diisopropylphosphoramidite-5'-dimethoxytrityl -5-(fur-2-yl)2'-deoxyuridine (3)

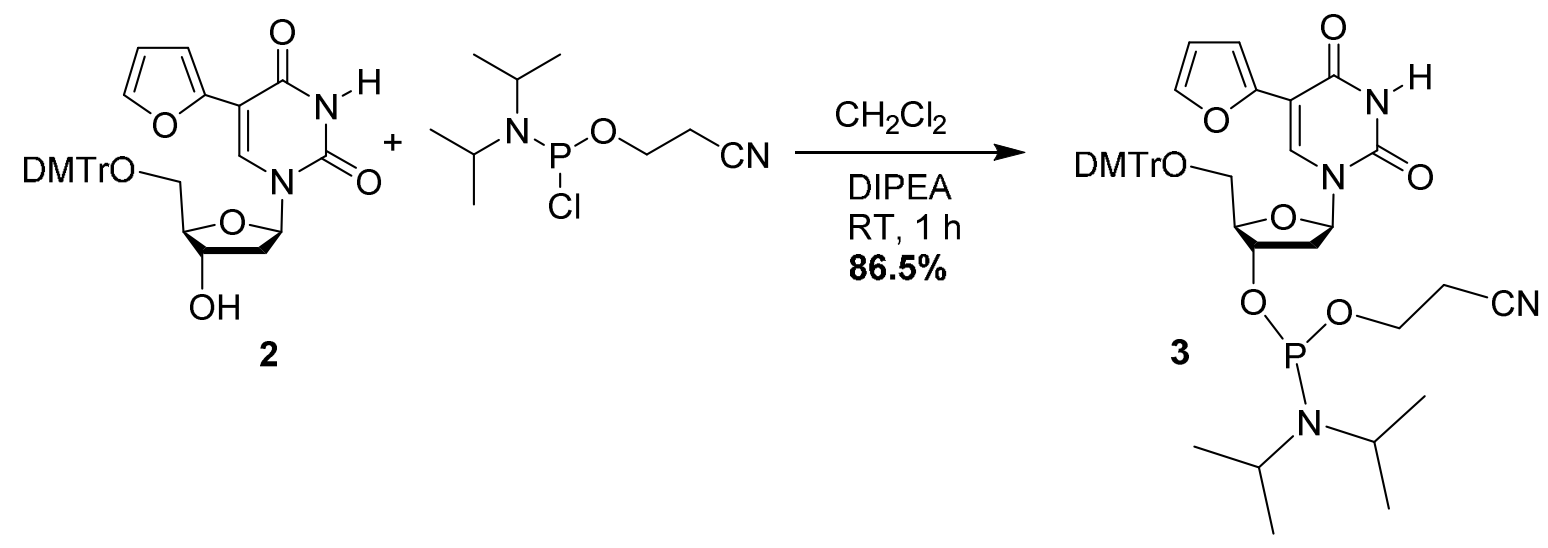


In a flame dried 50-mL RBF, 5'-Dimethoxytrityl-5-(fur-2-yl)-2'-deoxyuridine 2 (0.78 g, 1.3 $\mathrm{mmol}$ ) was dissolved in anhydrous $\mathrm{CH}_{2} \mathrm{Cl}_{2}(20 \mathrm{~mL})$ in the presence of molecular sieves $4 \AA$ after removal of the air from the flask under nitrogen atmosphere. The reaction mixture was stirred at room temperature under nitrogen atmosphere for 20 minutes. $\mathrm{N}, \mathrm{N}$-Diisopropylethylamine $(0.68 \mathrm{~mL}, 3.92 \mathrm{mmol})$ was added to the reaction mixture followed by 2-cyanoethyl- $N, N$-diisopropylchlorophosphoramidite $(0.5 \mathrm{~g}, 2.09 \mathrm{mmol})$ via a syringe. The reaction mixture was stirred at room temperature for $1 \mathrm{~h}$ (following the disappearance of the starting material by TLC; ethyl acetate/hexane; $1 / 1+1 \% \mathrm{Et}_{3} \mathrm{~N}$ ). The reaction mixture was diluted with $\mathrm{CH}_{2} \mathrm{Cl}_{2}$, filtered to remove the molecular sieves and concentrated under reduced pressure. The product was purified by flash column chromatography (1/1 ethyl acetate/hexanes, $\left.1 \% \mathrm{Et}_{3} \mathrm{~N}\right)$. The product was obtained as a light brown foam (0.9 g, 86.5\% yield). ${ }^{1} \mathrm{H}$ NMR (300 MHz, DMSO-d6): $\delta 11.60$ (s, NH, 1H), $7.98(\mathrm{~s}, 1 \mathrm{H}), 7.35(\mathrm{~d}, 2 \mathrm{H}), 7.24-7.15(\mathrm{~m}, 8 \mathrm{H}), 6.80-6.76(\mathrm{~m}, 5 \mathrm{H}), 6.43-6.41(\mathrm{~m}$, $1 \mathrm{H}), 6.14(\mathrm{t}, J=6.4 \mathrm{~Hz}, 1 \mathrm{H}), 4.45-4.43(\mathrm{~m}, 1 \mathrm{H}), 4.10-4.08(\mathrm{~m}, 1 \mathrm{H}), 3.66(\mathrm{~s}, 6 \mathrm{H})$, 3.58- $3.44(\mathrm{~m}, 4 \mathrm{H}), 3.20-3.15(\mathrm{~m}, 2 \mathrm{H}), 2.63-2.59(\mathrm{t}, J=6.6 \mathrm{~Hz}, 2 \mathrm{H}), 1.16-1.05(\mathrm{~m}$, $12 \mathrm{H}$ ); ${ }^{31} \mathrm{PNMR}\left(162 \mathrm{MHz}\right.$, DMSO-d6, referenced to $\mathrm{H}_{3} \mathrm{PO}_{4}$ ): $\delta 150.06,149.7$. 

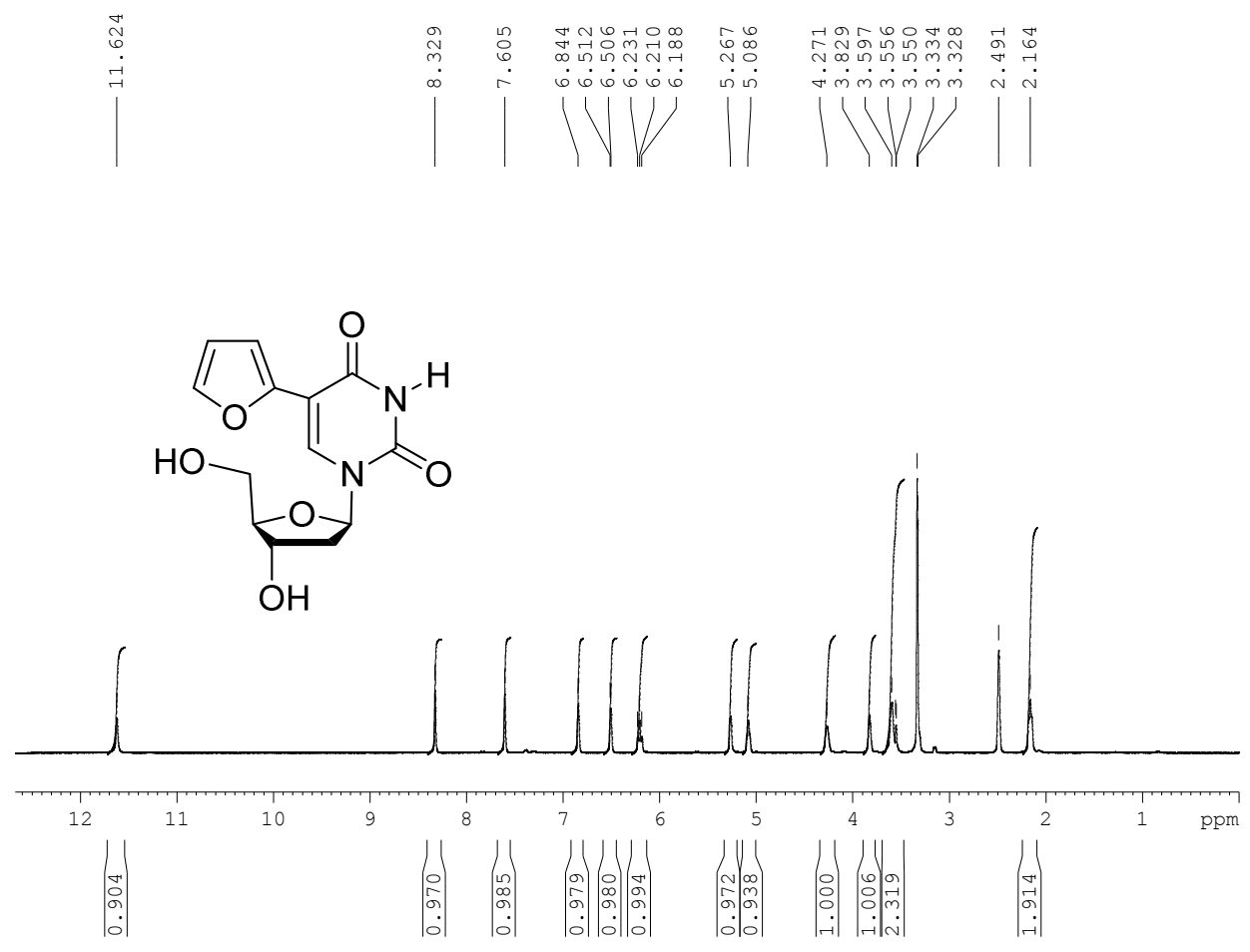

Figure S3. ${ }^{1} \mathrm{H}$ NMR spectrum of ${ }^{\mathrm{Fur}} \mathrm{dU}$ in DMSO- $\mathrm{d}_{6}$.

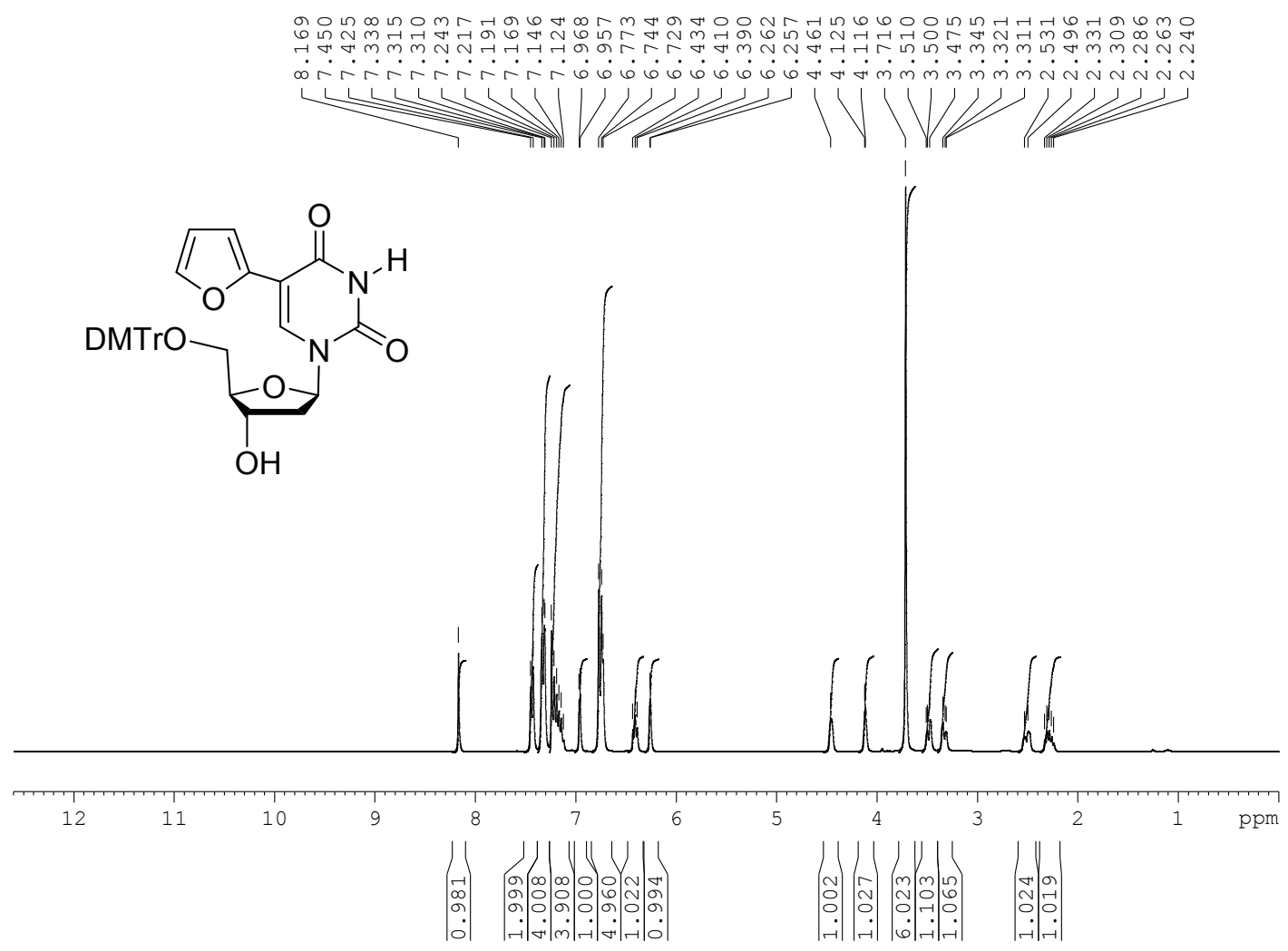

Figure S4. ${ }^{1} \mathrm{H}$ NMR spectrum of 2 in $\mathrm{CDCl}_{3}$. 


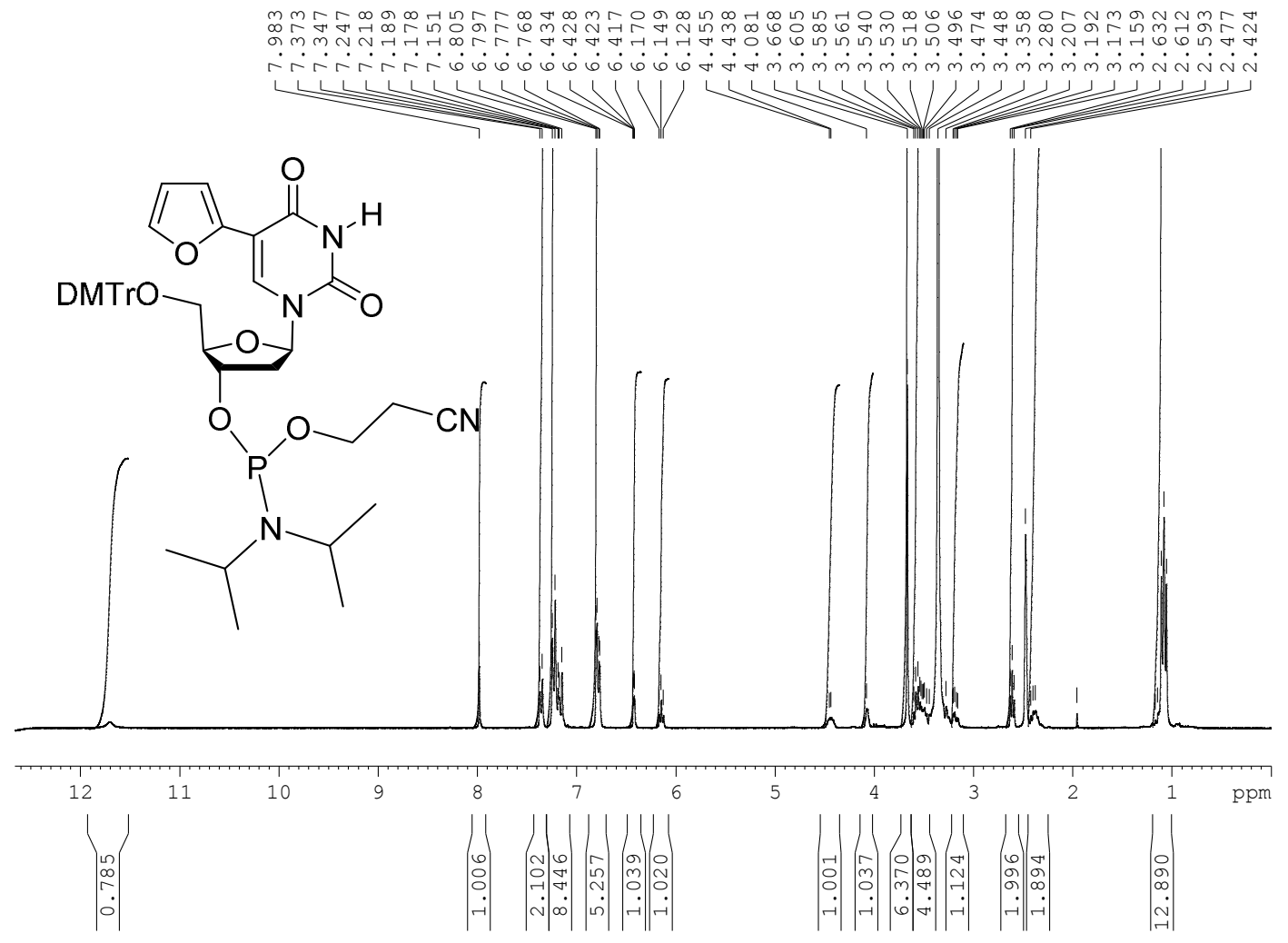

Figure S5. ${ }^{1} \mathrm{H}$ NMR spectrum of 3 in DMSO- $d_{6}$.
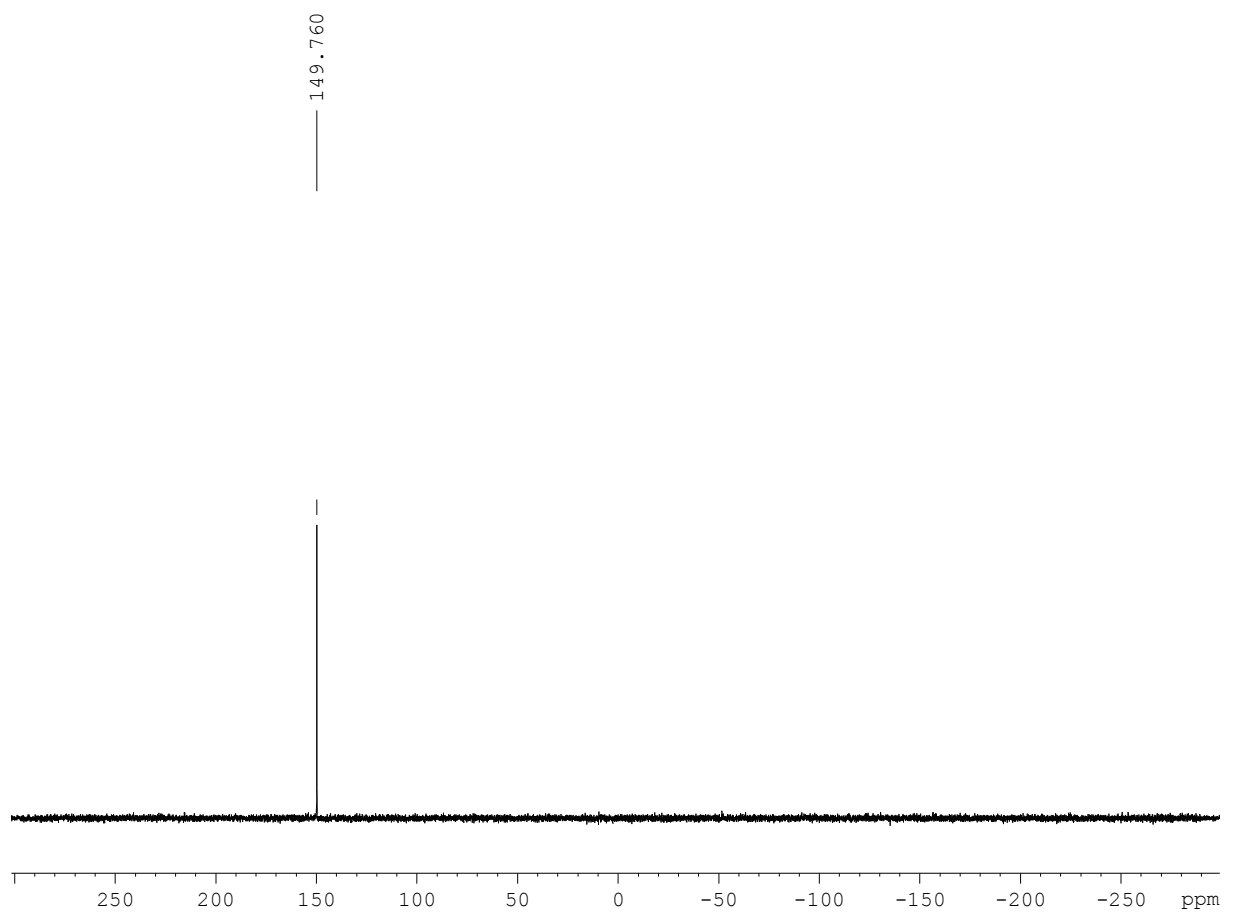

Figure S6. ${ }^{31} \mathrm{P}$ NMR spectrum of 3 in DMSO- $\mathrm{d}_{6}$. 
Table S1: Tabulated MS data of Fur dU mTBA strands.

\begin{tabular}{|c|c|c|c|c|}
\hline mTBA & Formula & Calc. Mass & $m / z(E S I)$ & Exp. Mass \\
\hline \multirow[t]{5}{*}{${ }^{\text {Fur }} \mathrm{dU}-3$} & $\mathrm{C}_{153} \mathrm{H}_{187} \mathrm{~N}_{57} \mathrm{O}_{95} \mathrm{P}_{14}$ & 4778.1 & {$[\mathrm{M}-9 \mathrm{H}]^{9-}=529.8$} & 4777.2 \\
\hline & & & {$[\mathrm{M}-8 \mathrm{H}]^{8-}=596.5$} & 4780.0 \\
\hline & & & {$[\mathrm{M}-7 \mathrm{H}]^{7-}=681.6$} & 4778.2 \\
\hline & & & {$[\mathrm{M}-6 \mathrm{H}]^{6-}=795.2$} & 4777.2 \\
\hline & & & {$[\mathrm{M}-5 \mathrm{H}]^{5-}=954.7$} & 4778.5 \\
\hline \multirow[t]{5}{*}{ FurdU-4 } & $\mathrm{C}_{153} \mathrm{H}_{187} \mathrm{~N}_{57} \mathrm{O}_{95} \mathrm{P}_{14}$ & 4778.1 & {$[\mathrm{M}-9 \mathrm{H}]^{9-}=530.1$} & 4779.9 \\
\hline & & & {$[\mathrm{M}-8 \mathrm{H}]^{8-}=596.4$} & 4779.2 \\
\hline & & & {$[\mathrm{M}-7 \mathrm{H}]^{7-}=681.7$} & 4778.9 \\
\hline & & & {$[\mathrm{M}-6 \mathrm{H}]^{6-}=795.4$} & 4778.4 \\
\hline & & & {$[\mathrm{M}-5 \mathrm{H}]^{5-}=954.9$} & 4779.5 \\
\hline \multirow[t]{5}{*}{ FurdU-7 } & $\mathrm{C}_{153} \mathrm{H}_{187} \mathrm{~N}_{57} \mathrm{O}_{95} \mathrm{P}_{14}$ & 4778.1 & {$[\mathrm{M}-9 \mathrm{H}]^{9-}=530.1$} & 4779.9 \\
\hline & & & {$[\mathrm{M}-8 \mathrm{H}]^{8-}=596.2$} & 4777.6 \\
\hline & & & {$[\mathrm{M}-7 \mathrm{H}]^{7-}=681.8$} & 4779.6 \\
\hline & & & {$[\mathrm{M}-6 \mathrm{H}]^{6-}=795.5$} & 4779.0 \\
\hline & & & {$[\mathrm{M}-5 \mathrm{H}]^{5-}=954.3$} & 4776.5 \\
\hline \multirow[t]{5}{*}{ FurdU-9 } & $\mathrm{C}_{153} \mathrm{H}_{187} \mathrm{~N}_{57} \mathrm{O}_{95} \mathrm{P}_{14}$ & 4778.1 & {$[\mathrm{M}-9 \mathrm{H}]^{9-}=530.1$} & 4779.9 \\
\hline & & & {$[\mathrm{M}-8 \mathrm{H}]^{8-}=596.4$} & 4779.2 \\
\hline & & & {$[\mathrm{M}-7 \mathrm{H}]^{7-}=681.7$} & 4778.9 \\
\hline & & & {$[\mathrm{M}-6 \mathrm{H}]^{6-}=795.1$} & 4776.6 \\
\hline & & & {$[\mathrm{M}-5 \mathrm{H}]^{5-}=954.7$} & 4778.5 \\
\hline \multirow[t]{5}{*}{ FurdU-12 } & $\mathrm{C}_{153} \mathrm{H}_{187} \mathrm{~N}_{57} \mathrm{O}_{95} \mathrm{P}_{14}$ & 4778.1 & {$[\mathrm{M}-9 \mathrm{H}]^{9-}=529.8$} & 4777.2 \\
\hline & & & {$[\mathrm{M}-8 \mathrm{H}]^{8-}=596.4$} & 4779.2 \\
\hline & & & {$[\mathrm{M}-7 \mathrm{H}]^{7-}=681.5$} & 4777.5 \\
\hline & & & {$[\mathrm{M}-6 \mathrm{H}]^{6-}=795.2$} & 4777.2 \\
\hline & & & {$[\mathrm{M}-5 \mathrm{H}]^{5-}=954.8$} & 4779.0 \\
\hline \multirow[t]{5}{*}{${ }^{\text {Fur dU-13 }}$} & $\mathrm{C}_{153} \mathrm{H}_{187} \mathrm{~N}_{57} \mathrm{O}_{95} \mathrm{P}_{14}$ & 4778.1 & {$[\mathrm{M}-9 \mathrm{H}]^{9-}=530.2$} & 4780.8 \\
\hline & & & {$[\mathrm{M}-8 \mathrm{H}]^{8-}=596.4$} & 4779.2 \\
\hline & & & {$[\mathrm{M}-7 \mathrm{H}]^{7-}=681.8$} & 4779.6 \\
\hline & & & {$[\mathrm{M}-6 \mathrm{H}]^{6-}=795.1$} & 4776.6 \\
\hline & & & {$[\mathrm{M}-5 \mathrm{H}]^{5-}=954.5$} & 4777.5 \\
\hline
\end{tabular}




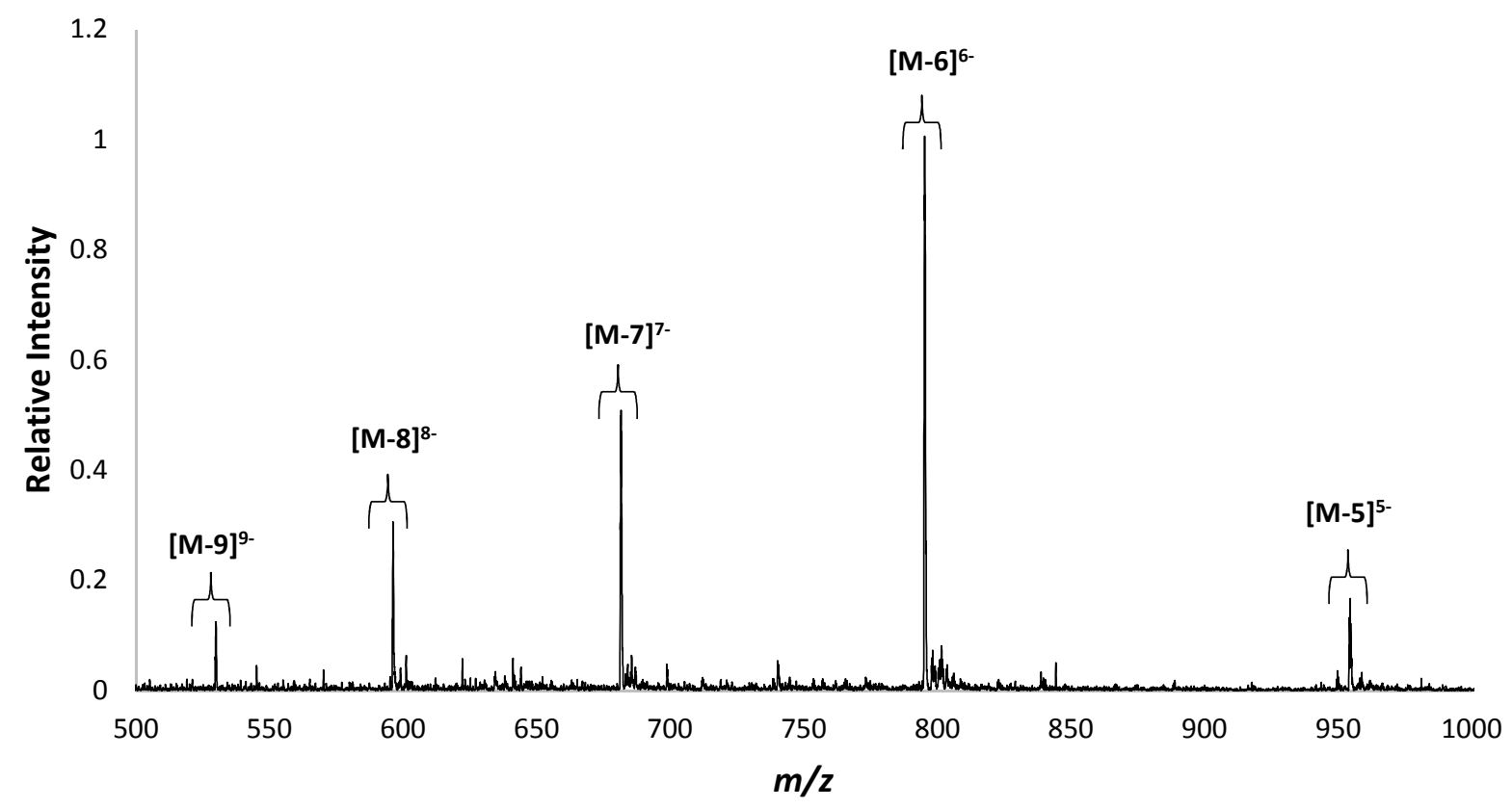

Figure S7. Representative $\mathrm{ESI}^{-} \mathrm{MS}$ Spectrum of ${ }^{\mathrm{Fur}} \mathrm{dU}-\mathrm{mTBA}$. 